\title{
ZUSCHRIFTEN
}

\section{Molekülstruktur und spektroskopische Eigenschaften des Kekulens ${ }^{[1]}$}

Von Claus Krieger, François Diederich, Dieter Schweitzer und Heinz A. Staab ${ }^{[*]}$

Kekulen (1), über dessen Synthese kürzlich berichtet wur$\mathrm{de}^{[2]}$, ist das erste Beispiel einer neuen Klasse aromatischer

I*J Prof. Dr. H. A. Staab. Ing. (grad.) C. Krieger, Dr. F. Diederich Abteilung Organische Chemie

Dr. D. Schweitzer

Abteilung Molekulare Physik

Max-Planck-Institut für medizinische Forschung

Jahnstraße 29, D-6900 Heidelberg 1
Verbindungen, bei der eine Anellierung von Sechsringen zu einem cyclischen System führt, das im Innern einen Hohlraum mit Wasserstoffatomen umschließt. Bei (1), für das sich 200 Kekulé-Strukturen mit unterschiedlicher Anordnung von Doppel- und Einfachbindungen formulieren lassen ${ }^{[3\}}$, interessierte die $\pi$-Elektronendelokalisation und die damit zusammenhängende Frage nach der Diatropie im makrocyclischen System, für die schon 1951 gezeigt worden war, daß verschiedene theoretische Ansätze im Falle von (1) zu kontroversen Voraussagen führen ${ }^{|4|}$. Experimentell ergab die ${ }^{1} \mathrm{H}$-NMR-Absorption der inneren Wasserstoffatome keine Anhaltspunkte für eine Diatropie im makrocyclischen $\mathrm{Sy}$ stem $^{[2]}$, was qualitativ mit MO-Berechnungen der Chemischen Verschiebungen übereinstimmt ${ }^{[4.5]}$. Im Zusammenhang mit diesen Fragen und einigen spektroskopischen Eigenschaften war die Bestimmung der Molekülstruktur des Kekulens durch Röntgen-Strukturanalyse von besonderem Interesse.
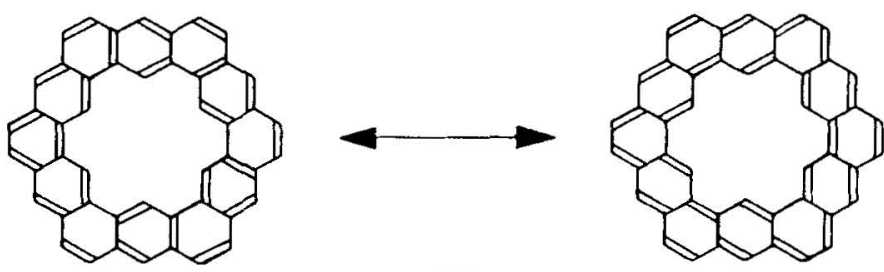

(1)

(1) wurde aus durch Zonenschmelzen gereinigtem Pyren in einer unter Hochvakuum geschlossenen Ampulle beim langsamen Abkühlen von 450 auf $350^{\circ} \mathrm{C}$ kristallisiert; nach Absublimieren des Pyrens im Hochvakuum und Waschen der Kristalle mit Chloroform erhielten wir gelbe monokline Nadeln, die sich für eine Strukturanalyse eigneten [Kristallgröße $0.05 \times 0.08 \times 0.4 \mathrm{~mm}$; Raumgruppe C2/c; $a=2799(3)$, $b=458.7(5), c=2271(2) \mathrm{pm}, \beta=109.6(1)^{\circ}, Z=4, \rho_{\mathrm{ber}}=1.45$ $\mathrm{g} \cdot \mathrm{cm}^{-3} ; \quad \mathrm{Mo}_{\mathrm{K} \alpha \mathrm{x}}-$ Strahlung, 1596 beobachtete Reflexe, $\mathbf{R}=0.056]$. 


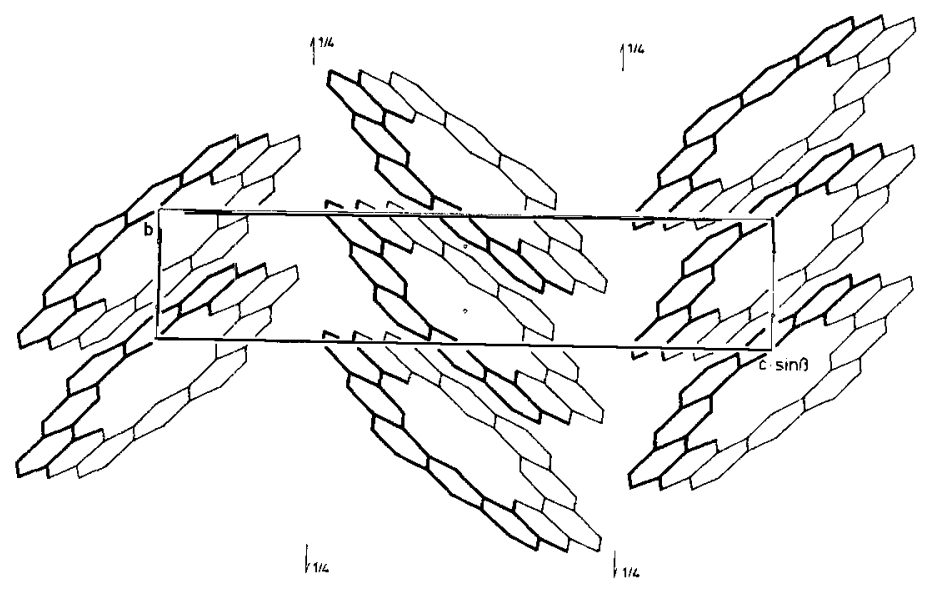

Abb. 1. Gitterstruktur von ( $I$ ) in der Projektion entlang der $a$-Achse (die hintere Hälfte der Elementarzelle wurde nicht gezeichnet).

Im Kristallgitter von (1) sind die Moleküle entlang der $b$ Achse gestapelt, wobei die Stapelachse mit den Molekülebenen einen Winkel von $42.9^{\circ}$ bildet. Moleküle, die innerhalb eines solchen Stapels benachbart sind, haben einen interplanaren Abstand von $336 \mathrm{pm}$ und sind um $312 \mathrm{pm}$ gegeneinander parallelverschoben. Die Molekülebenen benachbarter Stapel sind um $86^{\circ}$ gegeneinander geneigt, so daß sich in der Seitenansicht entlang der $a$-Achse das in Abbildung 1 gezeigte „Fischgrät-Gitter“ ergibt.

Mit einer mittleren Abweichung der Kohlenstoffatome von der mittleren Ebene durch die 48 Kohlenstoffatome von nur $3 \mathrm{pm}$ und einer maximalen Abweichung von $7 \mathrm{pm}$ hat (1) eine nahezu ideale ebene Struktur. Dies gilt auch für die sechs inneren Wasserstoffatome, für die die entsprechenden Abweichungen 5 bzw. $10 \mathrm{pm}$ betragen, obwohl hier die nicht-bindenden Abstände zwischen benachbarten Wasserstoffatomen mit 196(2) pm ungewöhnlich kurz sind.

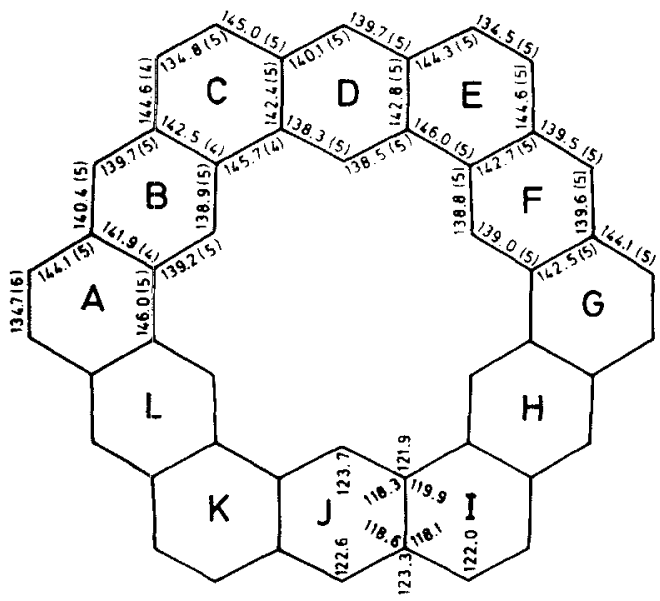

Abb. 2. Bindungslängen von (1) in pm (in Klammern Standardabweichungen in Einheiten der letzten angegebenen Stelle) und Valenzwinkel (als Mittelwerte äquivalenter Winkel).

Abbildung 2 macht deutlich, daß hinsichtlich der Bindungslängen zwischen den beiden Sechsring-Gruppen $A, C$, E, G, I, K und B, D, F, H, J, L klar zu unterscheiden ist: Nur in der zuletztgenannten Gruppe findet man mit Mittelwerten von $138.8 \mathrm{pm}$ für die Bindungen des inneren (1)-Perimeters und von $139.8 \mathrm{pm}$ für die Bindungen des äußeren Perimeters „,normale“ Arenbindungslängen, gegenüber denen allerdings alle sechs radialen Bindungen mit einem Mittelwert von $142.3 \mathrm{pm}$ signifikant verlängert sind. Drastische Abweichungen von den Bindungslängen dieser ,,aromatischen Ringe“
(B, D, ...) findet man jedoch bei der zweiten Gruppe von sechs äquivalenten Sechsringen (A, C, ...): Die sechs peripheren $\mathrm{CH}$.CH-Bindungen dieser Ringe haben mit einem Mittelwert von $134.6 \mathrm{pm}$ nahezu die Länge einer normalen C C-Doppelbindung, und dementsprechend haben die Bindungen zwischen diesen „Doppelbindungen" und den ,aromatischen Ringen“ ( $B, D, \ldots)$ mit einem Mittelwert von $144.5 \mathrm{pm}$ einen relativ hohen Einfachbindungscharakter. Noch stärker sind mit rund $146 \mathrm{pm}$ die Bindungen aufgeweitet, die im inneren Perimeter die ,,aromatischen Ringe" verbinden und dementsprechend dem Charakter der Einfachbindungen in Polyphenyl-Systemen nahekommen.

Angesichts der Zahl von 200 formulierbaren KekuléStrukturen für (1) muß die beträchtliche Bindungslokalisation, die aus den Bindungslängen hervorgeht, zunächst überraschen. Die beobachtete Abstufung der Bindungslängen wurde jedoch im qualitativen Trend - wenn auch nicht in dem tatsächlich gefundenen Ausmaß - aus MO-SCF-Berechnungen vorausgesagt ${ }^{[6]}$. $\mathrm{Zu}$ einer ausgezeichneten Übereinstimmung mit den experimentell bestimmten Bindungslängen führt die Auswertung der Summe der Kekulé-Strukturen in bezug auf die Paulingschen Bindungsordnungen der einzelnen Bindungen in $(1)^{171}$. Aufgrund der Strukturanalyse ist für das Kekulen zweifellos die Formulierung (1a) in der Clarschen Sextett-Schreibweise die beste Repräsentation der tatsächlich vorliegenden Bindungsverhältnisse.

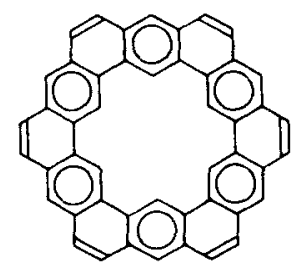

$|1 a|$

Mit der Sextett-Formulierung (1a) stimmt auch eine Reihe von spektroskopischen Eigenschaften des Kekulens überein: Das Elektronenspektrum von (1) zeigt eine auffallend kurzwellige Absorption, wie sie generell nach $\mathrm{Clar}^{\left[{ }^{[\beta}\right.}$ bei Aromaten des ,kondensierten Polyaryl-Typs“ wie z. B. beim Hexabenzocoronen gefunden wird (Abb. 3). Die symmetrie-verbotene $\alpha$-Bande von (1) ist zweifellos unter dem langwelligen Absorptionsanstieg der p-Bande verborgen; diesem $\alpha$-Übergang entspricht aber offenbar die stark schwingungs-strukturierte Fluoreszenzbande bei $453 \mathrm{~nm}$ (Abb. 4a).

Ebenso wie Hexabenzocoronen bei tiefer Temperatur eine starke Phosphoreszenz bei $575 \mathrm{~nm}$ (in 1,2,4-Trichlorbenzol)

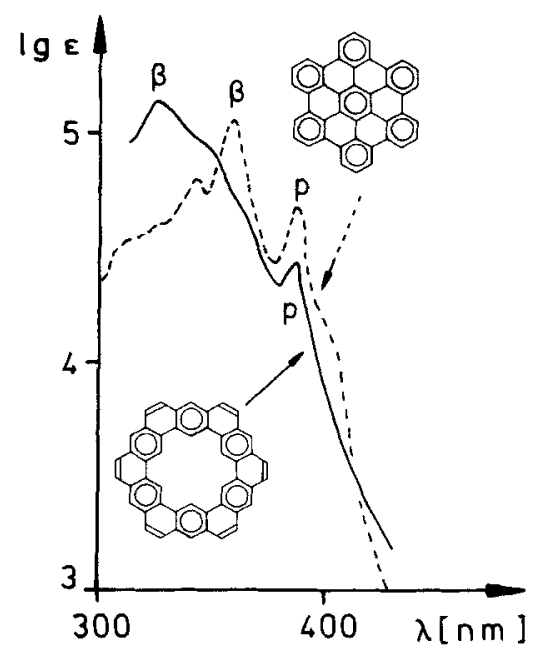

Abb. 3. Absorptionsspektren von (t) und Hexabenzocoronen (in 1,2,4-Trichlorbenzol). 
zeigt $^{\left[{ }^{[3]}\right.}$, beobachtet man bei (1) in $\left[\mathrm{D}_{2}\right]-1,2,4,5$-Tetrachlorbenzol-Matrix bei $1.3 \mathrm{~K}$ eine starke Phosphoreszenz mit mehreren im Bereich 585-595 nm eng beieinander liegenden, ,site"-abhängigen Maxima und einer Schwingungsstruktur, die auf die Kombination mit Molekülschwingungen im Bereich $1280-1630 \mathrm{~cm}^{-1}$ zurückzuführen ist (Abb. 4b).
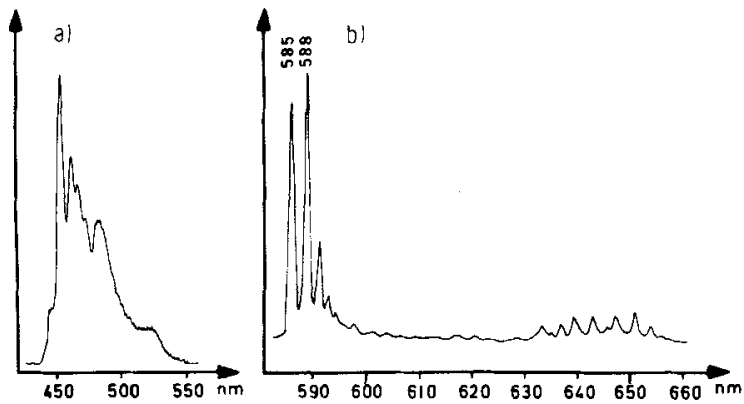

Abb. 4. a) Fluoreszenzspektrum von (1) (in 1,2,4-Trichlorbenzol, $298 \mathrm{~K}$, Anregung $326 \mathrm{~nm}$; b) Phosphoreszenzspektrum von $(\ell)$ (in $\left[\mathrm{D}_{2}\right]-1,2,4,5$-Tetrachlorbenzol, 1,3 K. Anregung $365 \mathrm{~nm}$ ). Ordinate: willkürliche Einheiten.

Die Phosphoreszenz von (1) ermöglicht die Anwendung der ODMR-Methode zur Bestimmung der Nullfeldaufspaltungsparameter $|E|$ und $|D|$ des angeregten Triplettzustandes ${ }^{[9]}$. Für (1) in $\left[D_{2}\right]-1,2,4,5-$ Tetrachlorbenzol-Matrix ergab sich ein $|E|$-Wert von $0.001935 \mathrm{~cm}^{-1}$, der entsprechend der $D_{6 h}$-Symmetrie von (1) erwartungsgemäß sehr niedrig ist (Benzol: $|\mathrm{E}|=0.0064 \mathrm{~cm}^{-1}$ ). Überraschend war aber, daß der $|\mathrm{D}|$-Wert - der ein Maß für die dipolare Kopplung der Triplett-Elektronen und daher umgekehrt proportional der 3. Potenz des durchschnittlichen Abstands dieser Elektronen ist - trotz der weiten räumlichen Ausdehnung des $\pi$-Elektronensystems im Kekulen mit $|D|=0.10622 \mathrm{~cm}^{1}$ nur wenig gegenüber dem Benzol reduziert und sogar größer ist als die |D|-Werte von Naphthalin und Anthracen (vgl. Benzol 0.1581 , Naphthalin 0.0994, Anthracen $\left.0.0694 \mathrm{~cm}^{-1}\right)$. Diese auf den ersten Blick unerwartet geringe Reduktion des $|\mathrm{D}|-$ Wertes $^{\left[{ }^{[0]}\right.}$ bringen wir qualitativ in Zusammenhang mit der partiellen Kompartmentierung des $\pi$-Elektronensystems in (1), die durch die Röntgen-Strukturanalyse nahegelegt wird ${ }^{[11]}$.

Eingegangen am 31. Juli 1979 [Z 293]

[1] Konjugation in makrocyclischen Bindungssystemen, 30. Mitteilung. - 29. Mitteilung: H. A. Staab, U. E. Meissner, A. Gensler, Chem. Ber.. im Druck.

[2] F. Diederich, H. A. Staab, Angew. Chem. 90, 383 (1978); Angew. Chem. Int. Ed. Engl. 17, 372 (1978) - W Jenny, P. Baumgartner, R. Paoni (ISNAProc., Sendai/Japan, August 1970) haben ohne Angabe der ublichen Daten den Anspruch erhoben, Kekulen (I) - dort ,[12]Coronaphen" genannt schon 1970 dargestellt zu haben. Da andere Autoren (vgl. z. B. [3]) sich auf diese Behauptung beziehen, sind wir zu der Feststellung veranlaßt, daß die Genannten bis heute weder eine Darstellung noch irgendwelche Eigenschaften des Kekulens in der chemischen Literatur veröffentlicht haben. Sie haben zwar in späteren thematisch verwandten Arbeiten ihren Anspruch der Synthese von (I) nicht wiederholt, aber sie haben es auch unterlassen. diesen Anspruch zurückzunehmen.

[3] J. Aihara, Bull, Chem. Soc. Jpn. 49, 1429 (1976).

[4] R. McWeeny, Proc. Phys. Soc. London A 64, 261, 921 (1951); zit. Lit.

[5] G. Ege, H. Vogler, Theor. Chim. Acta 26, 55 (1972); H. Vogler, Tetrahedron Lett. 1979,$229 ; C$. Wilcox, persöntiche Mitteilung.

[6] G. Ege, H. Fischer, Tetrahedron 23, 149 (1967)

[7] Wir danken Prof. W. C. Herndon. University of Texas in El Paso, für Diskussionsbeiträ̀ge.

[8] E. Clar: Polycyclic Hydrocarbons, Vol. 2. Academic Press, New York/ Springer-Verlag Berlin 1964, S. 97.

[9] Zur ODMR-Methode vgl. die Literaturangaben bei $D$. Schweitzer, $K, H$. Hausser, V. Taglieber, H. A. Staab, Chem. Phys. 14, 183 (1976).

[10] Bezüglich der Bindungsverhältnisse in (1) ist interessant, daß ähnliche ODMR-Resultate auch bei Hexabenzocoronen und verwandten Aromaten des „Polyaryl-Typs" mit hoher Molekülsymmetrie erhalten wurden: $J$. Voitländer, persönliche Mitteilung.

[11] Versuch einer theoretischen Deutung: $\boldsymbol{H}$. Vogler, Symposium on Aromaticity, Dubrovnik, September 1979 